\title{
Absence of blocking, overshadowing, and latent inhibition in social enhancement of food preferences
}

\author{
BENNETT G. GALEF, JR., and PAULA J. DURLACH \\ McMaster University, Hamilton, Ontario, Canada
}

\begin{abstract}
A rat's preference for food of a given flavor can be substantially enhanced by allowing it to interact with a conspecific demonstrator that has recently eaten food of that flavor. The heuristic value of treating such socially induced enhancement of flavor preference as an instance of Pavlovian conditioning was examined in three experiments. Conceiving of the smell of the food as a conditional stimulus and other cues emanating from the demonstrator rat as an unconditional stimulus, we determined whether each of three common Pavlovian phenomena-blocking, overshadowing, and latent inhibition-would occur. Using experimental parameters that readily produce socially induced enhancement of flavor preference, none of the three Pavlovian phenomena were found.
\end{abstract}

After a naive Norway rat (an observer) interacts with a recently fed conspecific (a demonstrator), the observer exhibits an enhanced preference for whatever food its demonstrator has eaten (Galef, Kennett, \& Wigmore, 1984; Galef \& Wigmore, 1983). Analyses of such changes in food preference indicate that they are truly social phenomena. That is, the enhancement of food preferences depends on the observers' experiencing food-related cues within the context provided by the presence of a conspecific demonstrator, not on the simple exposure of the observer rats to food-related cues during interaction with their respective demonstrators (Galef, Kennett, \& Stein, 1985; Galef \& Stein, 1985; Heyes \& Durlach, 1990). For example, feeding naive rats a food does not enhance their later preference for that food, whereas allowing naive rats to interact with a conspecific that has eaten the same food does enhance their preference for it (Galef, 1989; Galef \& Whiskin, 1992). Similarly, interaction with a demonstrator rat that has eaten a food is more effective than actually eating that food in interfering with observer rats' later learning of an aversion to the food in question (Heyes \& Durlach, 1990).

One way to conceptualize such social enhancement of food preference in rats is to consider the odor of a food carried on a demonstrator rat as a conditional stimulus (CS), which, when experienced by an observer rat in contiguity with aspects of a conspecific (an unconditional stimulus, or US), results in the observer's exhibiting an increased preference for the CS. If it is useful to think

This research was supported by grants from the Natural Sciences and Engineering Research Board of Canada and McMaster University Research Board to B.G.G., Jr. We thank Elaine Whiskin for assistance in conducting the experiments and Mertice Clark for thoughtful comments on earlier drafts of the manuscript. Correspondence should be addressed to P. J. Durlach, Department of Psychology, McMaster University, Hamilton, ON, Canada L8S 4K1. of the odor of a food as a CS, the response to which is altered as a result of a Pavlovian-conditioning-like process occurring during social interaction, then manipulations of the CS and US that have been found to interfere with association formation in more familiar examples of Pavlovian conditioning should similarly interfere with the social enhancement of responses to foods.

In the present series of experiments, we determined whether three well-documented Pavlovian conditioning phenomena-blocking (Kamin, 1969), overshadowing (Kamin, 1969; Pavlov, 1927), and latent inhibition (Lubow, 1973)-could be found in situations in which social induction of food preference is easily demonstrated.

\section{EXPERIMENT 1}

Experiment 1 was undertaken to determine whether either blocking (Kamin, 1969) or overshadowing (Kamin, 1969; Pavlov, 1927) occurs in situations in which the social enhancement of preference has been observed. Overshadowing refers to the finding that when a CS $\left(\mathrm{CS}_{1}\right)$ is paired with a US, less conditioned value accrues to $\mathrm{CS}_{1}$ if, at the time of pairing with the US, $\mathrm{CS}_{1}$ is accompanied by some other stimulus $\left(\mathrm{CS}_{2}\right)$ than if $\mathrm{CS}_{1}$ alone is paired with the US. Blocking refers to the finding that the interfering effect of $\mathrm{CS}_{2}$ on the conditioning of $\mathrm{CS}_{1}$ is increased, if $\mathrm{CS}_{\mathbf{2}}$ has had a history of pairing with the US (Kamin, 1969).

In Experiment 1, observer rats in overshadowing and blocking conditions interacted with demonstrator rats that had recently consumed a diet containing two distinct flavors $\left(\mathrm{CS}_{1}\right.$ and $\left.\mathrm{CS}_{2}\right)$. The observer rats in the blocking condition had each interacted on the previous day with a demonstrator rat that had eaten a diet containing only one of these flavors $\left(\mathrm{CS}_{2}\right)$. Of interest was the strength of the preference induced in response to $\mathrm{CS}_{1}$ in rats in 
the overshadowing and blocking conditions, in comparison with the preference induced in response to $\mathrm{CS}_{1}$ in rats in a conditioning group that had interacted with a demonstrator that had eaten a diet flavored with only $\mathrm{CS}_{1}$.

\section{Method}

\section{Subjects}

Seventy-two 42-day-old female Long-Evans rats, born in the vivarium of the McMaster University Psychology Department to breeding stock acquired from Charles River Canada (St. Constant, Quebec), served as observers. An additional seventy-two, 49- to 56-day-old female rats from the same source, which had served as observers in other experiments, served as demonstrators in the present experiment.

\section{Apparatus}

Both demonstrators and observers were housed throughout the experiment in individual wire-mesh hanging cages $(22 \times 24 \times$ $27.5 \mathrm{~cm}$ ). While in these cages, subjects were given ad-lib access to both water and food. The latter was presented to the subjects in semicircular (5-cm-diam) cups attached to one wall of each animal's cage.

\section{Diets}

The diets used in the experiment were made by adding various flavorants to powdered Purina Rodent Laboratory Chow 5001 (Diet Pur). Cinnamon-flavored diet (Diet Cin) was made by adding $1.0 \mathrm{~g}$ of McCormick's Fancy Ground Cinnamon to $100 \mathrm{~g}$ of Diet Pur, cocoa-flavored diet (Diet Coc) by adding $2.0 \mathrm{~g}$ of Hershey's Pure Cocoa to $100 \mathrm{~g}$ of Diet Pur, marjoram-flavored diet (Diet Mar) by adding $2.0 \mathrm{~g}$ of bulk ground marjoram to $100 \mathrm{~g}$ of Diet Pur, and cinnamon/marjoram-flavored diet (Diet $\mathrm{Cin} / \mathrm{Mar}$ ) by adding both $1.0 \mathrm{~g}$ of McCormick's Fancy Ground Cinnamon and $2.0 \mathrm{~g}$ of bulk ground marjoram to $100 \mathrm{~g}$ of Diet Pur.

\section{Procedure}

To begin the experiment, each demonstrator rat was placed on a 23-h food-deprivation schedule, eating Diet Pur for $1 \mathrm{~h} /$ day for 2 consecutive days. During this 2-day period, the observers were each maintained on pellets of Purina Rodent Laboratory Chow 5001, their familiar maintenance diet.

After this point in the procedure of Experiment 1, the subjects in each of the eight groups in the experiment were treated differently.

Conditioning groups. Following a third 23-h deprivation period, the demonstrators assigned to interact with the subjects in two conditioning groups were fed either Diet Cin (Experiment 1A) or Diet Mar (Experiment 1B) for $1 \mathrm{~h}$, and they were then placed, for $30 \mathrm{~min}$, in the cages of their respective observers. At the end of the 30-min period of interaction, the demonstrators were removed both from the cages of the observers and from the experiment. The observers were then offered a choice, for $22 \mathrm{~h}$, either between Diet Cin and Diet Coc (Experiment 1A) or between Diet Mar and Diet Coc (Experiment 1B).

Overshadowing groups. The subjects assigned to two overshadowing groups were treated exactly as were the subjects assigned to conditioning groups, except that the demonstrators that interacted with the observers assigned to overshadowing groups ate Diet $\mathrm{Cin} / \mathrm{Mar}$ when the demonstrators that interacted with the observers assigned to conditioning groups ate either Diet Cin (Experiment 1A) or Diet Mar (Experiment 1B).

Blocking groups. The subjects assigned to two blocking groups were treated exactly as were the subjects assigned to the overshadowing groups, except that each observer assigned to a blocking group interacted with its demonstrator for $.5 \mathrm{~h}$ on each of two occasions: first, after its demonstrator had eaten either Diet Mar (Experiment $1 \mathrm{~A}$ ) or Diet Cin (Experiment 1B) for $1 \mathrm{~h}$, and again, $24 \mathrm{~h}$ later, immediately after its demonstrator had eaten Diet Cin/Mar for $1 \mathrm{~h}$.

Control groups. The subjects assigned to the two control groups were treated exactly as were the subjects assigned to the conditioning groups, except that the demonstrators that interacted with the observers assigned to control groups ate either Diet Mar (Experiment 1A) or Diet Cin (Experiment 1B) when the demonstrators assigned to the conditioning group ate either Diet Cin (Experiment 1A) or Diet Mar (Experiment 1B). In other words, the demonstrators in the control groups consumed a flavor that was not presented to their observers during subsequent choice testing.

\section{Results and Discussion}

The results of Experiments $1 \mathrm{~A}$ and $1 \mathrm{~B}$ are presented in the two panels of Figure 1. As can be seen in Figure 1, in both Experiment 1A and Experiment $1 \mathrm{~B}$ there was an effect of treatment condition on the food choices of observers [Experiment 1A, $F(3,20)=4.38, p<.02$; Experiment $1 \mathrm{~B}, F(3,20)=9.58, p<.001]$. In both Experiments $1 \mathrm{~A}$ and $1 \mathrm{~B}$, the subjects in the conditioning, blocking, and overshadowing groups ate significantly
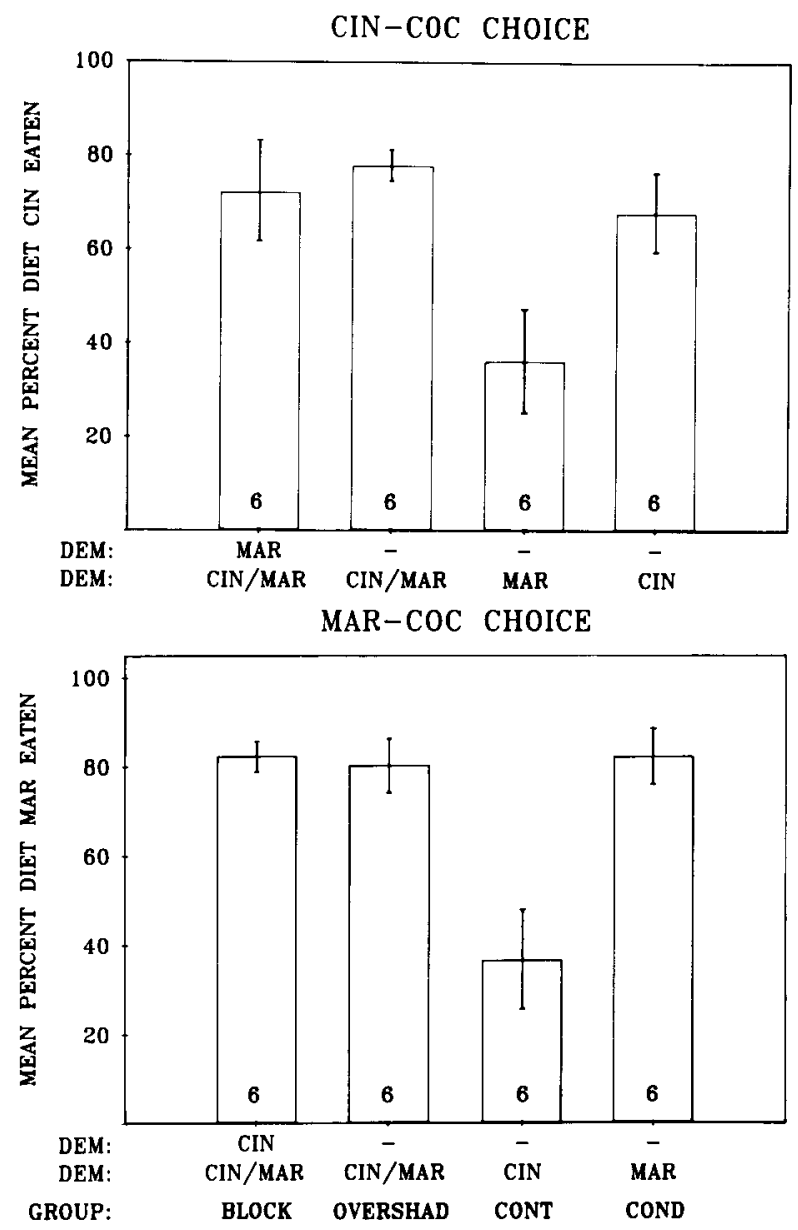

Figure 1. Mean amount of Diet Cin (upper panel) or Diet Mar (lower panel) eaten by observers in blocking (block), overshadowing (overshad), control (cont), and conditioning (cond) groups of Experiment 1. Numbers in histograms $=n$ /group. Flags $= \pm 1$ SEM. 
more Diet Cin (Experiment 1A) or Diet Mar (Experiment 1B) than did the subjects in their respective control groups (Tukey's tests, all $p s<.05$ ), but they did not differ from one another in intake of Diet Cin (Experiment 1A) or of Diet Mar (Experiment 1B) (Tukey's tests, $(p=$ n.s. in all instances).

Thus, although the results of Experiments 1A and 1B provided evidence of social enhancement of food preference (conditioning vs. control groups), the results of neither study provided evidence of either blocking or overshadowing effects in social enhancement of food preference (conditioning vs. blocking groups or conditioning vs. overshadowing groups).

\section{EXPERIMENT 2}

It is possible that the food-odor CSs used in Experiment 1 would not show blocking or overshadowing effects, regardless of the type of US with which they were paired (cf. Durlach \& Rescorla, 1980). To examine this possibility, in Experiment 2 we paired the food-odor CSs that we had used in Experiment 1 with an aversioninducing US. Galef, Wigmore, and Kennett (1983) have found that, after an observer rat both interacts with a recently fed conspecific demonstrator and receives an intraperitoneal injection of $\mathrm{LiCl}$ solution, the observer exhibits an aversion to whatever food its demonstrator has eaten. In the present experiment, we used this aversive conditioning procedure to determine whether food-odor CSs carried on demonstrators, CSs that did not exhibit overshadowing in Experiment 1, would exhibit overshadowing when paired with $\mathrm{LiCl}$-induced illness.

\section{Method \\ Subjects \\ Forty-eight 42-day-old female Long-Evans rats served as observers, and an additional forty-eight 56-day-old female Long- Evans rats that had served as observers in previous experiments served as demonstrators.}

Apparatus and Diets

The apparatus and diets were those used in Experiment 1.

\section{Procedure}

The procedure of the present experiment was similar to that used with the conditioning and overshadowing groups in Experiment 1. To begin the experiment, each demonstrator rat was placed on a 23-h deprivation schedule of eating Diet Pur for $1 \mathrm{~h} /$ day for 2 consecutive days. During this 2-day period, the observers were maintained on their familiar maintenance diet, Purina Rodent Laboratory Chow 5001, in pellet form.

Conditioning groups. Following a third 23-h deprivation period, the demonstrators assigned to interact with the observers in each of the two conditioning groups were fed either Diet Cin (Experiment 2A) or Diet Mar (Experiment 2B) for $1 \mathrm{~h}$, and they were then placed, for $30 \mathrm{~min}$, in the cages of their respective observers. At the end of the 30 -min period of interaction, the demonstrators were removed both from the cages of the observers and from the experiment. At the same time, the observers were injected i.p. with a $1 \mathrm{ml} / 100 \mathrm{~g}$ of body weight, $2 \% \mathrm{wt} / \mathrm{vol} \mathrm{LiCl}$ solution. Fifteen minutes following injection, each observer was offered a choice, for $22 \mathrm{~h}$, either between Diet Cin and Diet Coc (Experiment 2A) or between Diet Mar and Diet Coc (Experiment 2B).
Overshadowing groups. The observers in the two overshadowing groups were treated identically to the observers in the conditioning groups, except that their demonstrators were each fed Diet $\mathrm{Cin} / \mathrm{Mar}$ rather than Diet Cin (Experiment 2A) or Diet Mar (Experiment 2B) before they interacted with their respective observers.

Control groups. The observers in the control groups were treated identically to the observers in the overshadowing groups, except that they were injected with $1 \mathrm{ml} / 100 \mathrm{~g}$ of body weight of saline solution rather than $\mathrm{LiCl}$ solution.

\section{Results and Discussion}

The main results of Experiment 2 are presented in Figure 2, which shows the mean amount of Diet Cin (Experiment 2A) or Diet Mar (Experiment 2B) eaten by the observers in the conditioning, overshadowing, and control groups, as a percentage of the total amount that these observers ate during testing. As is clear from examination of Figure 2, the observers in the three groups in Experiment $2 \mathrm{~A}$ differed in percentage intake of Diet Cin $[F(2,18)=6.80, p<.007]$; the observers in the conditioning group ate less Diet Cin than did the observers in
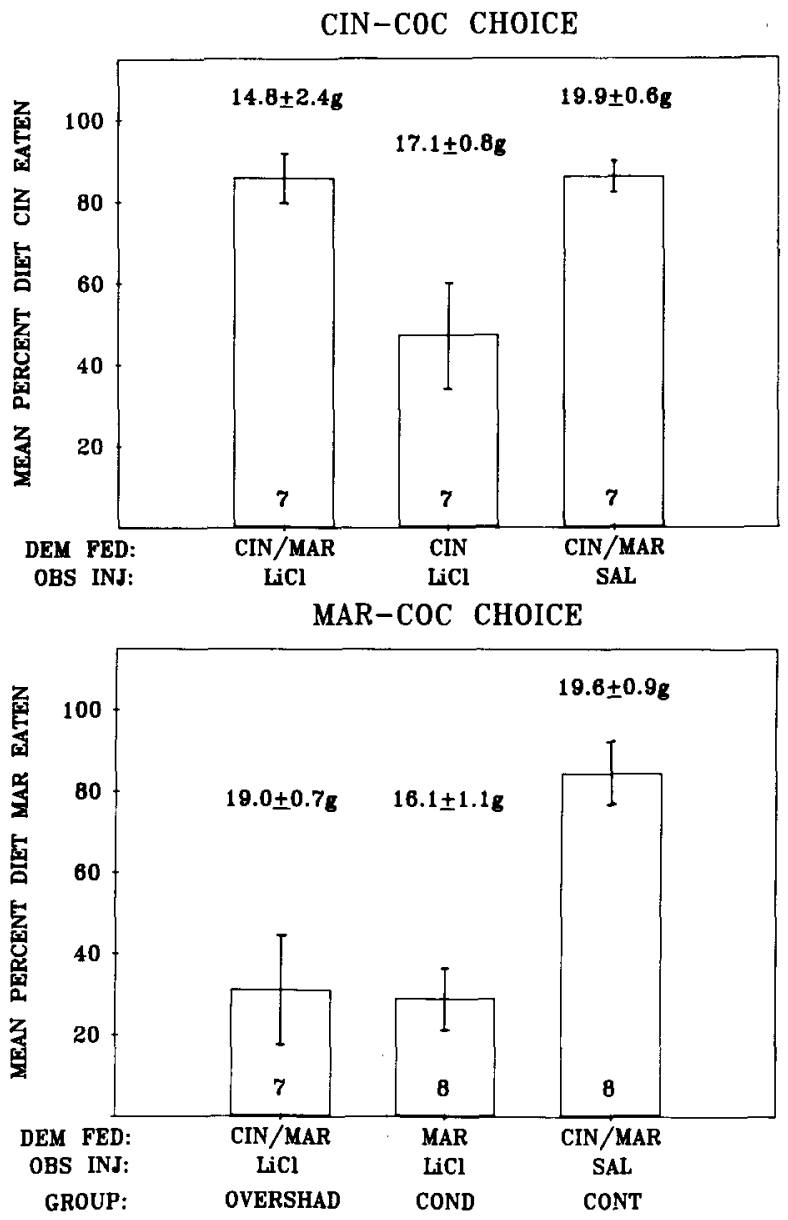

Figure 2. Mean amount of Diet Cin (upper panel) or Diet Mar (lower panel) eaten by observers in overshadowing (overshad), conditioning (cond), and control (cont) groups of Experiment 2. Numbers above histograms $=$ mean \pm 1 SEM of the total amount eaten during testing by observers in each group. Numbers in histograms = n/group. Flags $= \pm 1$ SEM. 
either the overshadowing or the control groups (Tukey's tests, both $p$ s <.01). Similarly, in Experiment 2B, there was a significant group effect on percentage intake of Diet $\operatorname{Mar}[F(2,21)=11.18, p<.001] ;$ the observers in the control group ate more Diet Mar than did the observers in either the conditioning or the overshadowing groups (Tukey's tests, both $p s<.05$ ). The presence of marjoram in the Diet Cin fed to the demonstrators in Experiment 2A completely overshadowed a learned aversion to Diet Cin; the presence of cinnamon in the Diet Mar fed to demonstrators in Experiment 2B failed to overshadow a learned aversion to Diet Mar.

Such a result is not unusual. Often when there is strong overshadowing of one CS $\left(\mathrm{CS}_{1}\right)$ by a second $\mathrm{CS}\left(\mathrm{CS}_{2}\right)$, little or no overshadowing of $\mathrm{CS}_{2}$ by $\mathrm{CS}_{1}$ is observed (Mackintosh, 1976). Thus, the results of Experiment 2 provide no support for the hypothesis that our failure to find evidence of overshadowing in Experiment 1 was the result of the nature of the CSs used in that experiment. On the contrary, the results of Experiments $2 \mathrm{~A}$ and $2 \mathrm{~B}$ indicate that food odors carried on a demonstrator rat can produce overshadowing when associated with an aversive US.

\section{EXPERIMENT 3}

The novelty of potential CSs is an important variable in determining the rate at which classical conditioning proceeds. Usually, the conditioning of a familiar CS occurs more slowly than the conditioning of an unfamiliar CS (Lubow \& Moore, 1959). This phenomenon is known as latent inhibition (Lubow, 1973). It might, therefore, be predicted that if social enhancement of food preference depends on classical conditioning involving a foododor CS, then extensive preexposure to that food CS would produce latent inhibition that interferes with subsequent conditioning.

In a previous experiment, Galef (1989) fed rats both a cinnamon-flavored diet (Diet $C$ in) and a cocoa-flavored diet (Diet Coc) ad lib for 7 days before allowing each rat to interact with a conspecific demonstrator fed either Diet Cin or Diet Coc. Even after 7 days of preexposure to both Diet Cin and Diet Coc, interaction with a demonstrator still produced, in the observers, a robust preference for the food eaten by their demonstrators (Galef, 1989). However, Galef's experiment was not designed to explore the effects of CS preexposure on socially induced flavor preference. Consequently, there were no control groups to permit the evaluation of the magnitude of any stimulus preexposure effects on the social enhancement of food preferences. We undertook the present experiment to directly explore the effects of flavor preexposure on socially induced flavor preferences in Norway rats.

\section{Method}

Subjects

One hundred and sixty 42 day-old female Long-Evans rats born in the vivarium of the McMaster University Psychology Depart- ment to breeding stock acquired from Charles River Canada (St. Constant, Quebec) served as observers in the present experiment. One hundred and twenty-eight additional 60- to 74-day-old rats from the same source, which had served as subjects in other experiments. served as demonstrators in the present experiment.

\section{Apparatus and Diets}

The apparatus and diets used in Experiment 3 were the same as those used in Experiments 1 and 2.

\section{Procedure}

Habituation. The observers and demonstrators were placed in individual wire-mesh hanging cages $(22 \times 24 \times 27.5 \mathrm{~cm})$, where they were maintained on ad-lib food (pellets of Purina Rodent Laboratory Chow 5001) and water for 2 days before the start of the experimental procedures.

Stimulus preexposure. At the end of the 2-day period of habituation, all food pellets were removed from each subject's home cage, and each subject was fed, for 24 h/day, Diet Cin, Diet Coc, or both Diet Cin and Diet Coc. In the last case, Diet Cin and Diet Coc were presented in separate containers. The subjects were preexposed to diets either for 1 day (the 1-day preexposure group) or for 3 days (the 3-day preexposure group).

Social induction of preference. At the end of the period of stimulus exposure, all food was removed from the observers' cages and a single demonstrator rat was placed for $30 \mathrm{~min}$ in the cage of each of 128 of the observers.

Seventy-two hours before these demonstrators were introduced into their respective observers' cages, each had been placed on a 23-h deprivation schedule and fed Diet Pur for $1 \mathrm{~h}$ both 49 and $25 \mathrm{~h}$ before being introduced into the observers' home cages. For the $1 \mathrm{~h}$ immediately before the demonstrators were placed in the observers' home cages, 64 of the demonstrators were fed Diet Cin and the remaining 64 were fed Diet Coc.

Thirty-two additional subjects, assigned to four control groups ( $n=8$ /group), were left undisturbed in their home cages during the 30 min when their fellows were interacting with demonstrators.

Testing observers. At the end of the 30 -min period of interaction between demonstrators and observers, the demonstrators were removed both from the cages of the observers and from the experiment. The 160 observers were then each offered, for $22 \mathrm{~h}$, two weighed food cups, one containing Diet $\mathrm{Cin}$ and the other containing Diet Coc.

\section{Results}

The results of Experiment 3 are presented in Figures 3 and 4 , which show, respectively, the mean amount of Diet Cin, as a percentage of total amount eaten, ingested during testing by the observers in the 1-day (Figure 3) and 3-day (Figure 4) preexposure conditions.

To simplify discussion of the results of Experiment 3, we have statistically analyzed the data from the subjects in the 20 groups of the experiment as though they belonged to six separate experiments, as is indicated by the vertical partitions dividing both Figure 3 and Figure 4 into three panels.

\section{One-Day Preexposure}

As can be seen in the left-hand panel of Figure 3, and as statistical tests confirmed, 1 day of preexposure to both Diet Cin and Diet Coc did not influence the subsequent effect of the interaction with demonstrators fed either Diet Cin or Diet Coc on the observers' later diet preferences. 


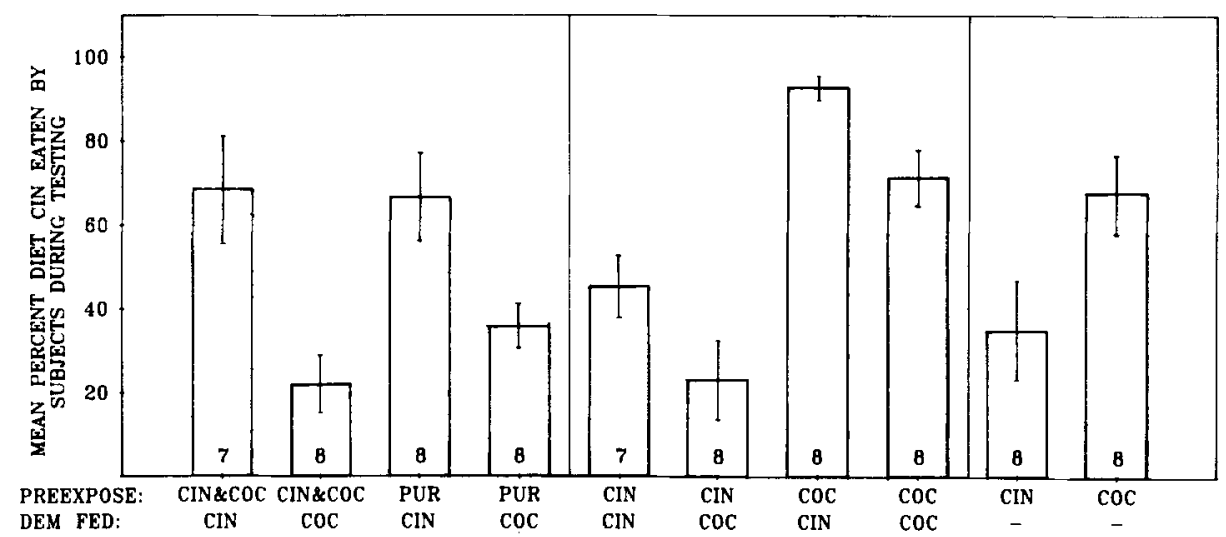

Figure 3. Mean amount of Diet Cin eaten by observers during testing following 1 day of preexposure and interaction with a demonstrator. $\mathrm{Cin}=$ Diet $\mathrm{Cin}, \mathrm{Coc}=$ Diet $\mathrm{Coc}, \mathrm{Dem}=$ demonstrator. Numbers in histograms $=n$ /group. Flags $= \pm 1 S E M$.

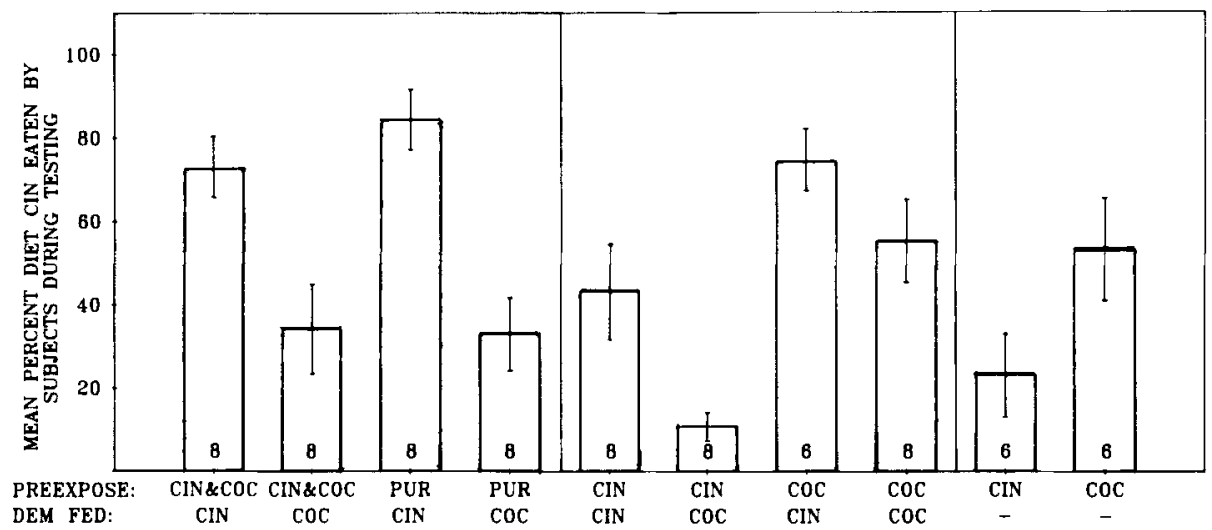

Figure 4. Mean amount of Diet Cin eaten by observers during testing after 3 days of preexposure and interaction with a demonstrator. Cin $=$ Diet Cin, Coco $=$ Diet Coc, Dem $=$ demonstrator. Numbers in histograms $=$ n/group. Flags $= \pm 1$ SEM.

A $2 \times 2$ analysis of variance (ANOVA) revealed a significant effect of the diet fed to demonstrators on their observers' food choices during testing $[F(1,28)=27.14$, $p<.001]$, but no effect of 1 day of preexposure to Diets Cin and Coc on the observers' food choices during testing $[F(1,28)=0.362, p=.55]$ and no significant interaction $[F(1,28)=.562$, n.s. $]$. This result confirms the finding of Galef (1989) that preexposure of observer rats to both of the diets that they are to choose between during testing does not significantly influence the size of subsequent demonstrator influence on observer preference for one of those diets.

The central panel of Figure 3 provides data describing the effect of preexposure to either Diet Cin or Diet Coc on later demonstrator-induced enhancement of preference for those diets. A $2 \times 2$ ANOVA revealed significant effects of preexposure diet $[F(1,26)=7.96, p<.009]$ and demonstrators' diet $[F(1,26)=8.49, p<.007]$ on the observers' diet preferences during subsequent testing, but no significant interaction $[F(1,26)=.68$, n.s. $]$.
Regardless of which food the demonstrators ate, the observers preexposed to Diet Cin ate less Diet Cin during testing than did the observers preexposed to Diet Coc. Regardless of preexposure diet, the observers that interacted with the demonstrators fed Diet Cin ate more Diet Cin during testing than did the observers that had interacted with the demonstrators fed Diet Coc.

Examination of the right-hand panel of Figure 3 provides additional weak evidence of effects of prior exposure to diets on the food preferences of observers: The observers preexposed to Diet Coc for 1 day before testing ate marginally more Diet Cin during testing than did the observers preexposed to Diet Cin for 1 day before testing ( $t=3.69, p=.08$ ).

\section{Three-Day Preexposure}

The effects of 3 days of preexposure to diets paralleled, but were larger than, those of 1-day preexposure to diets. As can be seen in the left-hand panel of Figure 4, we found, once again, that preexposure to both Diet Cin and 
Diet Coc had no effect $[F(1,27)=.43, p>.51]$ on subsequent enhancement by demonstrators of their observers' diet preferences $[F(1,27)=17.7, p<.0003]$.

On the other hand, as can be seen in the central panel of Figure 4, preexposure to either Diet Cin or Diet Coc had a massive effect $[F(1,27)=44.8, p<.00001]$ on the observers' later diet preferences, as did the diet fed to the demonstrators $[F(1,27)=9.28, p<.006]$. Post hoc Tukey's tests revealed that preexposure of an observer for 3 days to the diet fed to its demonstrator significantly reduced the magnitude of the demonstrators' effects on their observers' food preferences during testing (Tukey's tests: Cin, Coc vs. Coc,Coc, $p<.01$; Coc, Cin vs. Cin, Cin, $p<.01$ ).

Finally, as can be seen in the right-hand panel of Figure 4, preexposing subjects to either Diet Cin or Diet Coc for 3 days produced a significant enhancement of preference for the other diet during testing $(t=4.72, p<.05)$.

\section{Discussion}

The results of Experiment 3 indicate that prolonged preexposure of observer rats to diets directly affects their preferences for preexposed diets. However, the data provide little evidence that diet preexposure interferes directly with the acquisition of socially induced preferences. Preexposing observers to both Diet Cin and Diet Coc for 3 days had no effect on subsequent socially induced preferences for either Diet Cin or Diet Coc. Preexposing observers either to Diet Cin or to Diet Coc reduced their preference for the preexposed diet. Preexposure appeared to detract from the strength of the socially induced preference for a diet, not by changing the associability of the preexposed taste, but, instead, by inducing a tendency to avoid eating the preexposed food.

We were, of course, surprised to find that exposure to either Diet Cin or Diet Coc reduced the preference for that diet when it was later available concurrently with a diet of novel flavor. From the literature (Corey, 1978; Hill, 1978), we had expected that eating a diet would cause an enhancement of the preference for that diet as opposed to a diet that was completely novel. Such enhanced preference for a relatively familiar diet would have altered preference in the same direction as did social learning. Consequently, any latent inhibition effect would have been measured against two factors enhancing the preference for the preexposed diet.

In contrast to our expectations, diet preexposure decreased preferences, independently of any subsequent social learning experience. Because the decrease in preferences due to preexposure and the increase in preferences due to social learning appeared simply to summate, the results provided no evidence that latent inhibition influenced social learning.

\section{GENERAL DISCUSSION}

The present series of experiments was undertaken to determine whether experimental procedures that often interfere with Pavlovian conditioning would interfere with the induction of socially enhanced flavor preferences. In Experiment 1, we failed to find evidence of either blocking or overshadowing in the social enhancement of flavor preferences. In Experiment 3, we failed to find evidence of latent inhibition of the social enhancement of flavor preferences.

Because there may have been something about the particular CSs used in Experiment 1 (food odors carried on a demonstrator rat) that made them particularly resistant to overshadowing regardless of the US with which they were associated, we performed Experiment 2, in which the CSs used in Experiment 1 were used as CSs in a conditioned aversion paradigm. We found convincing evidence of overshadowing of the odor of cinnamon by the odor of marjoram when these food odors carried on a demonstrator rat were followed by illness.

It is not clear why we found evidence of overshadowing in Experiment 2 but not in Experiment 1; perhaps, different processes were enlisted by social and toxic USs. Perhaps, differences in the two experiments in the representation of the CS at the time of US occurrence caused differences in outcome. For example, if the smell of the food eaten by a demonstrator is viewed as a CS, and if some aspect of the demonstrator itself is viewed as a US, then, in Experiment 1, the CS and US were experienced simultaneously by observers. In Experiment 2, on the other hand, an olfactory CS and a toxic US were presented to observers sequentially. Consequently, the occurrence of overshadowing in Experiment 2A might have been the result of some change in representation of the compound CS during the time interval between the CS and US, rather than the result of a difference in associability of elements of the compound CS with the different USs used in Experiments 1 and 2 .

It may have been unduly optimistic to hope to find overshadowing, blocking, and latent inhibition in our experimental paradigms. Overshadowing and blocking, although they are considered to be general phenomena, are not entirely reliable, even in traditional Pavlovian paradigms (Dickinson, Nicholas, \& Mackintosh, 1983; Durlach \& Rescorla, 1980; Williams, 1981). Furthermore, we used only a single compound-stimulus trial to assess blocking and overshadowing in the social enhancement of food preferences, whereas both blocking and overshadowing have been discussed frequently as multitrial phenomena (Mackintosh, 1975; Mackintosh, Bygrave, \& Picton, 1977; Rescorla \& Wagner, 1972). On the other hand, both overshadowing and blocking have been demonstrated with the use of a single compound-stimulus trial (Balaz, Kasprow, \& Miller, 1982; Dickinson et al., 1983; James \& Wagner, 1980; Mackintosh \& Reese, 1979), and we did find evidence of overshadowing when we used a single compound-stimulus trial in an aversive-conditioning situation (Experiment 2). Latent inhibition, although a robust phenomenon (Lubow, 1989), can be context specific (Kaye, Preston, Szabo, Druiff, \& Mackintosh, 1987). So, for example, it is possible that eating a food and experienc- 
ing the odor of that food on a demonstrator were sufficiently different modes of presentation that any loss of associability resulting from eating a food during preexposure failed to transfer to the social learning situation.

It might also be argued that the Pavlovian paradigm is inappropriate for analysis of the social enhancement of food preference. We cannot clearly specify the nature of the US, and we also have some difficulty in defining both conditioned and unconditioned responses. Still, Pavlovian analyses have proven fruitful in the interpretation of studies of taste-aversion learning (Domjan, 1980), in which there are similar problems in precisely defining stimuli and responses (Rozin \& Kalat, 1972).

The failure to find evidence of overshadowing, blocking, or latent inhibition in our studies was, therefore, disappointing. We had hoped that the demonstration of such effects would provide an avenue for further exploration of the behavioral processes supporting the social enhancement of flavor preferences. The results of the experiments reported here do not suggest that, in studies of the social enhancement of food preference, treating food odors and demonstrator rats as conditional and unconditional stimuli will prove as heuristic as has the similar treatment of flavors and toxicosis in studies of flavor-aversion learning.

It is, of course, always possible that, with the exploration of a wider set of experimental parameters, Pavlovian phenomena such as overshadowing, blocking, and latent inhibition could be demonstrated in settings where socially induced enhancement of food preferences occurs. However, the mechanisms underlying such Pavlovian effects appear to have little influence under a set of conditions in which the basic social learning effect is easy to obtain.

\section{REFERENCES}

Balaz, M. A., Kasprow, W. J., \& Miller, R. R. (1982). Blocking with a single compound trial. Animal Learning \& Behavior, 10, 271-276.

COREY, D. T. (1978). The determinants of exploration and neophobia. Neuroscience \& Biobehavioral Reviews, 2, 235-253.

Dickinson, A., Nicholas, D. J., \& Mackintosh, N. J. (1983). A reexamination of one-trial blocking in conditioned suppression. Quarterly Journal of Experimental Psychology, 35B, 67-79.

DomJan, M. (1980). Ingestional aversion learning: Unique and general processes. In J. S. Rosenblatt, R. A. Hinde, C. Beer, \& M. C. Busnel (Eds.), Advances in the study of behavior: Vol. 11 (pp. 276-337). New York: Academic Press.

Durlach, P. J., \& Rescorla, R. A. (1980). Potentiation rather than overshadowing in flavor aversion learning: An analysis in terms of within-compound associations. Journal of Experimental Psychology: Animal Behavior Processes, 6, 175-187.

GALEF, B. G., JR. (1989). Enduring social enhancement of rats' preferences for the palatable and the piquant. Appetite, 13, 81-92.

Galef, B. G., JR., Kennett, D. J., \& Stein, M. (1985). Demonstrator influence on observer diet preference: Effects of simple exposure and the presence of a demonstrator. Animal Learning \& Behavior, $13,25-30$
Galef, B. G., JR., Kennett, D. J., \& Wigmore, S. W. (1984). Transfer of information conceming distant foods in rats: A robust phenomenon. Animal Learning \& Behavior, 12, 292-296.

Galef, B. G., JR., \& STEIN, M. (1985). Demonstrator influence on observer diet preference: Analyses of critical social interactions and olfactory signals. Animal Learning \& Behavior, 13, 31-38.

GALEF, B. G., JR., \& WhiSKIN, E. E. (1992). Social transmission of information about multiflavored foods. Animal Learning \& Behavior, 20, 56-62.

Galef, B. G., JR., \& Wigmore, S. W. (1983). Transfer of information concerning distant foods: A laboratory investigation of the "information-centre" hypothesis. Animal Behaviour, 31, 748-758.

Galef, B. G., JR., Wigmore, S. W., Kennett, D. J. (1983). A failure to find socially mediated taste-aversion learning in Norway rats (R. norvegicus). Journal of Comparative Psychology, 97, 358-363.

Heyes, C. M., \& Durlach, P. J. (1990). "Social blockade" of taste aversion learning in Norway rats ( $R$. norvegicus): Is it a social phenomenon? Journal of Comparative Psychology, 104, 82-87.

HILL, W. (1978). The effects of mere exposure on preferences in nonhuman mammals. Psychological Bulletin, 85, 1177-1198.

JAMES, J. H., \& WAGNER, A. R. (1980). One-trial overshadowing: Evidence of distributed processing. Journal of Experimental Psychology: Animal Behavior Processes, 6, 188-205.

Kamin, L. J. (1969). Predictability, surprise, attention, and conditioning. In B. A. Campbell \& R. M. Church (Eds.), Punishment and aversive behavior (pp. 279-296). New York: Appleton-Century-Crofts.

Kaye, H., Preston, G. C., Szabo, L., DruifF, H., \& Mackintosh, N. J. (1987). Context specificity of conditioning and latent inhibition: Evidence for a dissociation of latent inhibition and associative interference. Quarterly Journal of Experimental Psychology, 39B, 127-145.

LuBow, R. E. (1973). Latent inhibition. Psychological Bulletin, 79, 398-407.

LuBow, R. E. (1989). Latent inhibition and conditioned attention theory. New York: Cambridge University Press.

LuBow, R. E., \& Moore, A. U. (1959). Latent inhibition: The effect of nonreinforced exposure to the conditioned stimulus. Journal of Comparative \& Physiological Psychology, 52, 415-419.

Mackintosh, N. J. (1975). A theory of attention: Variations in the associability of stimuli with reinforcement. Psychological Review, 82, 276-298.

MaCKINTOSH, N. J. (1976). Overshadowing and stimulus intensity. Animal Learning \& Behavior, 4, 186-192.

Mackintosh, N. J., Bygrave, D. J., \& Picton, B. M. (1977). Locus of the effect of a surprising reinforcer in the attenuation of blocking. Quarterly Journal of Experimental Psychology, 29, 327-336.

MaCkINTOSH, N. J., \& REESE, B. (1979). One-trial overshadowing. Quarterly Journal of Experimental Psychology, 31, 519-526.

Pavlov, I. P. (1927). Conditioned reflexes. London: Oxford University Press.

Rescorla, R. A., \& Wagner, A. R. (1972). A theory of Pavlovian conditioning: Variations in the effectiveness of reinforcement and nonreinforcement. In A. H. Black \& W. F. Prokasy (Eds.), Classical conditioning II: Current research and theory (pp. 64-99). New York: Appleton-Century-Crofts.

RozIN, P., \& KALAT, J. W. (1972). Learning as a situation-specific adaptation. In M. E. P. Seligman \& J. L. Hager (Eds.), Biological boundaries of learning (pp. 66-96). New York: Appleton-Century-Crofts.

Williams, B. A. (1981). Blocking in an autoshaping procedure. Behavior Analysis Letters, 1, 345-351.

(Manuscript received April 29, 1992; revision accepted for publication December 16, 1992.) 